VIII.

Aus dem pharmakologisehen Institnt der Universität Zürich.

\title{
Über die Wirkung der Hypnotika (Neuronal) bei normalen und bei psychisch erregten Zuständen.
}

Von

P. Gensler.

Die Veranlassung zu dieser Arbeit gab die allen Arzten und besonders den Psychiatern bekannte Tatsache, daß die Schlafmittel bei Aufregungszuständen von den Patienten in weit größeren Mengen als normalerweise vertragen werden.

Das angestrebte Ziel war, nach den Ursachen dieser Erscheinung zu forschen. Selbstverständlich konnte das Problem nicht auf einmal gelöst werden, aber ich versuchte wenigstens es an einem Punkte anzufassen, und zwar dort, wo es der objektiven Beurteilung am meisten zugänglich war. Ich bemühte mich daher, die quantitativen Verhältnisse zwischen Schlafmittel und Gehirnsubstanz sowohl im Normalen wie bei Aufregungszuständen festzustellen. Es sollte damit der Versuch gemacht werden, klarzulegen, ob die erwähnte Abschwäehung der Wirkung der Hypnotika vielleicht in einer Veränderung der Zellmembran etwa im Sinne einer Herabsetzung der Durehlässigkeit derselben und eines daraus resultierenden Widerstandes gegen das Mittel zu suchen sei, oder im funktionellen Zustand des Zellprotoplasmas selber liege. Man könnte sich für den letzteren Fall ja vorstellen, daß die Zelle bei Aufregungen in einem Zustande erhöhter Funktion sich befinde und deshalb das Narkotikum in den iblichen Mengen seine spezifische Wirkung nicht entfalten könne, etwa wie bei dem antagonistischen Verhalten Kurare: Physostigmin.

Als Versuchssubstanz wurde das Neuronal $\left(\mathrm{CBr}\left(\mathrm{C}_{2} \mathrm{H}_{5}\right)_{2} \mathrm{CONH}_{2}\right.$ $=$ Bromdiäthylacetamid) gewählt, das mir von der Firma Kalle \& Co. Archiv f. exporiment. Path. u. Pharmakol. Bd. 77 . 
in freundlicher Weise zur Verfügung gestellt wurde. Ich bin anf diese Substanz rerfallen, weil sie erstens als Schlafmittel sich gut eingefuhrt hat, zweitens wie die meisten Hypnotika die Eigenschaft besitzt, bei psychischen Erregtwgssuständen wenig zu wirken und drittens sich durch einen hohen Bromgehalt $(41,48 \%$ ) auszeichnet. Letzteres schien mir deshalb von Bedeutung, weil ich an Hand des Bromgehaltes der Organe, speziell des Gehirns, die dorthin gelangte Menge an Neuronal bestimmen wollte. Es ist dies die einzig zuverlässige Methode, um quantitativen Aufschluß über die Verteilung eines Schlafmittels zu erhalten, weil normalerweise das Gehira gar kein Brom enthält, das Neuronal sein Brom sehr schwer abspaltet und das rom Brom abgelöste Molekül keine narkotische Wirkung mehr besitzt.

Außerdem konnte gleichzeitig damit ein Beitrag zu der noch relativ wenig bekannten Pharmakologie des Nenronals erbracht werden. Angaben über das Verhalten desselben finden sich bei D. Eeckhouty, Krieger und v. d. Velden ${ }^{2}$ ). Experimentelles iuber Bromural, Neuronal und Adalin ist zu finden bei $\mathrm{S}$. Takeda ${ }^{3}$, J. $\mathrm{Kwan}{ }^{4}$ ).

Von besonderem Interesse für meine Fragestellung sind die beiden leteteren Arbeiten.

Takeda untersuchte speziell das Bromural, Kwan die Beziehungen von Bromural, Adalin und Neuronal zueinander. Sie konstatierten folgendes: Die Intensität der. Wirkung geht proportional dem Gehalte des Gehirns an unverändertem Bromural (Adalin und Neuronal). Eine Konzentration von 0,0083\% Bromural genuigt zur Narkose, von $0,0223 \%$ zum Tode. Die Zerstörung findet im Gehirn nur in kleinem Maße statt, mehr dagegen in der Leber und in den Muskeln. Das gleiche gilt für das Neuronal und das Adalin. Das Neuronal ist das wirkungsvollste, es erreicht die Hirnsubstanz am schnellsten und verweilt dort am längsten. Der Eintritt des Schlafes ist an eine gewisse Menge gebunden. Die molekulare Giftigkeit des Bromurals und Adalins sind ziemlich gleich, die des Neuronals ist schwächer. Die Verteilung ist bei allen dreien die gleiche, das Gehirn enthält am meisten nnzersetzte, die Leber am meisten zersetzte Substanz. Diese Versuche wurden an normalen Kaninchen ausgeführt. Dabei ist allerdings za bemerken, daß die Autoren zur quantitativen Brombestimmung sich einer kolorimetrischen Methode bedienten, die

1) Areh. f. exper. Path. u. Pharmakol. Bd. 57, S. 338, 1907.

2) Dentsch. med. Wochenschr. 1907, Nr. 6.

3) Archives internat. de Pharmakodynamie et de Thérapie XXI, S. 203, 1911.

4) Ebenda XXII, S. 331, 1912. 
mir nicht ganz einwandfrei rom chemischen Standpunkt ans erscheint. Trotzdem scheinen die erhaltenen Resultate ziemlich gleichmäßig.

Zu meinen Experimenten wurden nur Hunde verwendet. Kaninchen erschienen mir psychisch als viel zu indifferent für derartige Untersuchungen. Ferner sollten auch die Resorptionsbedingungen unter sich bei den einzelnen Tieren möglichst gleich und ähnlich denen beim Menschen sein. Ich verabreichte deshalb das Neuronal stets als Gummiemulsion in gleicher Konzentration und immer bei nüchternem Magen mit der Schlundsonde. Die Versuche werden zeigen, daß damit eine sehr gleichmäßige Resorptionsgeschwindigkeit erzielt wird.

Von den elf Hunden waren die mit A, E, G, J bezeichneten als Kontrollhunde bestimmt, um die quantitativen Verhältnisse im Gehirn und die Verteilung im Organismus im normalen Zustande klarzulegen. Bei den übrigen Tieren wurden pathologische Verhältnisse herbeigeführt, und zwar bei B, C, D, F, K und L durch Injektion eines Aufregungsmittels und bei $\mathrm{H}$ durch äußere Kunstgriffe und direkte psychische Beeinflussung.

Als Aufregungsmittel diente in fünf Fällen das Monomethyl des ac-Tetrahydro- $\beta$-Naphthylamin, in einem Falle die Base dieses Körpers. Über Chemie und Pharmakologie dieser Substanzen findet sich das Nähere bei Cloetta und Waser1). Diese Substanzen wurden in Mengen von $0,06-0,1 \mathrm{~g}$ subkutan injiziert.

Es wurde ferner streng darauf geachtet, daß bei jedem Tiere zwischen dem letzten Vorversuch und dem definitiven quantitativen Versuch ein Zeitraum von mindestens vier bis fünf Tagen lag. Hält man sich nicht an diese Regel, so kann es vorkommen, daß noch kleine Brommengen im Körper vorhanden sind, die sich unter Umständen auch ins Gehirn verirren können und die quantitative Neuronalanalyse daselbst illusorisch machen. Um genaue Vergleichswerte zu erhalten, wurden alle Tiere, deren Organe analysiert werden sollten, 2 Stunden nach der Eingabe des Neuronals getötet.

Zur Entnahme der Organe wurde in folgender Weise vorgegangen: Dem aufgebundenen Tiere werden links die Jugularis und die Carotis freigelegt, in die Vene wird eine Kanüle eingeführt, um die Spülflüssigkeit (isotonische und durch Gelatine isovisköse Ringerlösung) einlaufen zu lassen. Aus der Carotis wird zuerst das zur Analyse notwendige Blut $(2 \times 100 \mathrm{ccm})$ aufgefangen. Sobald dann

1) Arch. f. exper. Path. n. Pharmakol, Bd. 73, S. 398. 
die Hauptmenge des Blutes ausgelanfen ist, das Herz aber noch gut schlägt, läßt man langsam die Spülflüssigkeit einlaufen, bis das Herz nicht mehr arbeitet. Es findet so eine Ausspülung der Organe in streng physiologischer Weise ohne Quellungserscheinungen statt. Die Menge der verwendeten Flüssigkeit wurde immer der Größe des Tieres angemessen, erreichte aber auch beim größten Hunde nicht mehr als 2 Liter, so daß man kaum Gefahr lief, etwa Neuronal künstlich auszuschwemmen. Die Temperatur der Spülftüssigkeit betrug $37-38^{\circ} \mathrm{C}$. Es gelang auf diese Art jedesmal, praktisch blutleere Gehirne zu erhalten. Darauf wurden dem Tiere das Gehirn, ein abgewogenes Stuick Leber und der Magen- und Duodenalinhalt entnommen und mit der Blutportion quantitativ auf Neuronal analysiert.

Zur Feststellung des Gehaltes der Hirnsubstanz und der anderen Organe an Neurona] wurde, wie bereits erwähnt, das darin enthaltene Brom bestimmt.

Die Voruntersuchungen für die chemische Methodik dauerten ziemlich lange, weil einerseits das Material schwierig zu verarbeiten, andererseits an die Genauigkeit große Anforderungen zu stellen waren. Ich habe es zuerst mit der durch $\nabla$. Wys 1 ) ausgearbeiteten Methodik versucht. Diese besteht darin, daß das anorganische Brom aus den Organen extrahiert und dann das farblose Filtrat mit konzentrierter Schwefelsäure und Kaliumbichromat in einem Kolben leicht erwärmt wird, um das Brom zu vertreiben. Dieses leitet man in einem kräftigen Luftstrom durch eine Serie von Standgläsern, in welchen sich eine Jodkalilösung in verschiedenen Konzentrationen befindet. Das entweichende Jod wird mit Natriumthiosulfat titriert und daraus das Brom berechnet.

Diese Methode hat sich aber für die Bestimmung ron organisch gebundenem Brom nicht bewährt. Die Werte fielen immer um 20 bis $25 \%$ zu niedrig ans. Sehr genaue Ergebnisse erhielt ich dagegen nach folgendem Verfahren: Das entblutete Gehirn wird gleich in der Nickelschale zersttuckelt und gut mit etwas Ätzkali in Substanz vermischt, darauf getrocknet und vorsichtig verbrannt. Sodann zieht man die Kohle mit etwas heißem Wasser aus, dampft, obne zu filtrieren, ein und verascht. Nun wird die Asche in heißem Wasser anfgenommen und filtriert. Das Filtrat muß ganz klar und farblos sein, anderenfalls soll es nochmals verdampft und eventuell unter Zusatz von Salpeter verascht werden. Die farblose Lösung, die nun das organisiert

1) Arch. f. exper. Path. u. Pharmakol. Bd. 5o, S. 263. 
eingefuhbrte Brom als Bromkalium enthält, wird am besten unter Erwärmen mit Salz- oder Schwefelsäure neutralisiert oder leicht angesäuert, sodann mit Chlorwasser titriert. Das Chlorwasser verdrängt das Brom ans seiner anorganischen Verbindung, was eine Gelbfärbung der Lösung bedingt, und zwar so lange, als poch Brom enthalten ist. Näheres über diese Methode findet sich bei Treadwelli). Es empfieblt sich, das Filtrat möglichst einzuengen. Dadurch wird der Farbenumschlag deutlicher. Das Chlorwasser muß natürlich farblos and stark verdünnt sein.

Das Blut und die Leber wurden ganz gleich behandelt wie das Gehirn. Der Mageninhalt wurde mit etwas Kalilauge alkaliseh gemacht und eingedampft, dann in der Nickelschale mit etwas Ätzkali nud Substanz verascht. Die ganze Prozedur muß sehr vorsichtig ansgeführt werden, vor allem muß man jedes Glïhen vermeiden. Die Schalen wurden nie der direkten Flamme ansgesetzt, sondern immer uiber dem Asbestring erbitzt; besonders beim Blnt und bei großen Gehirnen mnßte öfters drei- bis viermal eingedampft und verascht werden, bis das Filtrat farblos war. Bei Beobachtung aller Vorsichtsmaßregeln beeinträchtigt dies die Analyse in keiner Weise, erfordert aber viel Zeit.

Die nach diesen Verfahren ansgeführten Kontrollanalysen ergaben sehr befriedigende quantitative Resultate. Von dem den Organen zugesetzten Neuronal wurden 99,7, 100 and 100,5\% wiedergefunden.

\section{Versuche.}

\section{Verguch. Hund A. 9. I. 14. 7,1 kg Gewicht.}

Da znerst. die schlafmachende Dosis ausprobiert werden mußte, erhielt der Hund in den nüchternen Magen 9,38 Ubr a. m. $3 \mathrm{~g}$ Neuronal $=0,42 \mathrm{~g}$ pro Kilogramm Gewicht.

Puls vor der Darreichung 120 Schläge, Atmung 20. Schon nach etwa 5 Minuten wird das Tier etwas unruhig, bald darauf stellen sich Koordinationstörungen ein und der Gang wird stark schwankend. Nach 10 Minuten legt sich das Tier nieder und verfällt bald in festen Schlaf.

$9,55 \mathrm{Uhr}$ a. m. ist es fest eingeschlafen.

Puls 180, Atmung 26; auf Kneifen und Stechen erfolgt keine Reaktion mehr. Der Kornealreflex ist erloschen und der Pnpillarreflex träge.

10,05 Uhr Atmung 26; Puls 180

10,20 Uhr Atmung 40; Puls 180 fester Schlaf.

10,30 Uhr Atmung 25; Puls 180

10,55 Uhr, also nach einer Stunde Schlaf, Atmung 30, Puls 170; zu den übrigen Erscheinungen gesellt sich ein heftiges Zittern und Zucken

1) Lehrbnch der Analyt. Chemie. 
des ganzen Körpers, besonders der Extremitäten, wohl ein Intoxikationssymptom. Dieser Zustand dauerte 60 Stunden an. Mit der Zeit ging der Puls auf etwa 90 Schläge herunter, die Atmung bewegte sich immer um 30 herum, die Sensibilitätsstörung blieb unverändert, ebenso das Zittern. Nach 62 Stunden erwachte das Tier, es dauerte aber noch einen ganzen Tag, bis es sich einigermaßen erholt hatte.

\section{Versuch. Hand A. 15. I. $14.6,2 \mathrm{~kg}$ Gewicht.}

Gestützt auf das Resultat des ersten Versuches, ging ich mit der Dosis auf $0,1 \mathrm{~g}$ pro Kilogramm Gewicht hinunter. 9,30 Uhr a. m. $0,1 \mathrm{~g}$ Nenronal pro Kilogramm Gewicht nüchtern mit der Sonde, total also $0,62 \mathrm{~g}$ Neuronal.

Nach 5 Minuten schwankender Gang, bald darauf legt sich das Tier nieder und ist

9,40 Uhr eingeschlafen. Puls 160; Atmung 24.

Stechen und Kneifen sind erfolglos, Kornealreflex erloschen. Das vorhin beschriebene Zittern stellt sich auch bald wieder ein, ist jedoch nicht so heftig.

10,00 Uhr Puls 145; Atmung 20.

10,20 Uhr Puls 145; Atmung 22.

10,40 Uhr Puls 150; Atmung 25.

11,10 Uhr Pals 150; Atmung 24.

11,40 Uhr Puls 150; Atmung 18.

Der Schlaf ist unverändert, auch das Zittern hält noch an.

2,00 Uhr p. m. erwacht das Tier, bleibt aber noch liegen, es zittert noch etwas. Puls 150; Atmung 25.

3,30 Uhr. Der Hund kann schon ganz gut gehen, schwankt nur sehr wenig und erscheint ganz munter.

3. Versuch. Hund A. 20. 1. 14. 6,4 kg Gewicht.

8,52 Uhr a. m. $0,1 \mathrm{~g}$ Neuronal pro Kilogramm, total also $0,64 \mathrm{~g}$. Es stellt sich wieder nach etwa 5 Minuten das Schwanken mit den Koordinationsstörungen ein.

9,00 Uhr schläft das Tier ein.

9,20 Uhr Puls 120; Atmung 12.

9,40 Uhr Puls 80: Atmung 12.

10,30 Uhr Puls 90; Atmung 13.

Das Zittern hat sich auch wieder eingestellt.

11,20 Uhr Puls 84; Atmung 12.

11,40 Uhr Puls 84; Atmung 12.

Dieser ruhige Schlaf dauert bis

2,00 Uhr p. m., dann erwacht das Tier und läuft nach einiger Zeit ganz munter und ziemlich sicher umher.

Da die beiden letzten Versuche mit je $0,1 \mathrm{~g}$ Neuronal pro Kilogramm Gewicht eine ziemlich übereinstimmende und regelmäßige Wirkung gezeigt haben, wurde zum letzten und definitiven Versuche mit diesem Tier geschritten. 
Wirkg.d.Hypnotika (Neuronal/ b. normal. u. b. psychisch erregten Zuständen. 167

\section{Versuch. Hund A. 26. I. 14. 6,5 kg Gewicht.}

$8,40 \mathrm{Uhr}$ a. m. $0,1 \mathrm{~g}$ Neuronal pro Kilogramm Gewicht, total $=0,65 \mathrm{~g}$. Die Wirkung zeigt sich nach der gewöhnlichen Zeit und in der üblichen Form, doch ist das Tier etwas unruhig.

9,00 Uhr schläft es ein.

10,40 Uhr, also 2 Stunden nach Darreichung des Mittels, wird der Hund nach der schon beschriebenen Methode getötet. Ausnahmsweise wurde hier noch schnell eine Messung des Blutdruckes vorgenommen, die einen Druck von $150 \mathrm{mg} \mathrm{Hg}$ ergab, somit eine völlig normale Zahl.

Die quantitative Analyse der Organe ergab:

Gewicht des Gehirns $6 \check{\mathrm{g}}$, Bromgehalt $5,5 \mathrm{mg}$. Auf $100 \mathrm{~g} 8,4 \mathrm{mg}$.

In Prozent des eingeführten Neuronals $2 \%$. Auf $100 \mathrm{~g} 3 \%$.

In $100 \mathrm{ccm}$ Blnt gefunden $32 \mathrm{mg}$ Brom. Auf $500 \mathrm{ccm}$ Blut berechnet $\left.160 \mathrm{mg}^{1}\right)$.

In Prozent des eingeführten Neuronals 60\%.

Als unresorbiert in Magen und Darm gefunden $45 \mathrm{mg}$ Brom.

In Prozent des eingefübrten Neuronals $16,5 \%$.

\section{Versuch. Hund B. 9. I. $14.15,2 \mathrm{~kg}$ Gewicht.}

2,43 Uhr p. m. erhält das Tier $0,25 \mathrm{~g}$ Neuronal pro Kilogramm Gewicht, total also $3,8 \mathrm{~g}$. Puls war 104; Atmung 18.

Es stellt sich auch hier nach 5-6 Minuten das Schwanken als Folge der Koordinationsstörungen ein.

2,56 Uhr tritt leichter Schlaf ein, doch ist das Tier noch zu wecken.

3,11 Uhr fester Schlaf. Atmung 22; Puls 160. Kornealreflex noch nicht ganz erloschen, Sensibilität sehr stark herabgesetzt, Sehnenreflexe erhöht. Auf heftige Reize reagiert das Tier aber noch.

3,23 Uhr Atmung 20; Puls 160; Kornealreflex erloschen, sonstiger Zustand unverändert.

4,11 Uhr Atmung 16; Puls 144.

5,11 Uhr Atmung 15; Puls 140.

Das'Zittern des Körpers hat sich auch hier eingestellt, doch nicht in so hohem Maße wie bei Tier A.

Dieser tiefe Schlaf dauerte 18 Stunden, 5-6 Stunden nach dem Erwachen hatte sich der Hund gut erholt und lief munter nmher.

\section{Versueh. Hund B. 11. I. 14. $14 \mathrm{~kg}$ Gewicht.}

Auch 0,25 g pro Kilogramm Gewicht war zu stark wirkend gewesen, deshalb erhielt der Hund, wie Hand A,

9,00 Uhr a. m. $0,1 \mathrm{~g}$ Nenronal pro Kilogramm Gewicht, total also $1,4 \mathrm{~g}$.

1) Die Blutmenge der Tiere wurde dadurch berechnet, daß man das Gewicht des Tieres durch 13 dividierte. Diese Art der Berechnung bringt es selbstverständlich mit sich, daß die Summe der Prozente bald höher bald niedriger als 100 ausfällt. 
Das Tier legt sich gleich nieder und ist

9,10 Uhr fest eingeschlafen.

9,15 Uhr Atmung 26; Puls 120; Sensibilität star's herabgesekzt, Kornealreflex sehr träge.

9,25 Chr a. m. Puls 108; Atmung 30.

9,40 Uhr a. m. Puls 84; Atmung 20.

9,55 Uhr a. m. Puls 84; Atmung 20.

10,10 Uhr a. m. Puls 82; Atmung 17.

10,40 Uhr a. m. Puls 70; Atmung 13.

11,10 Uhr a. $\mathrm{m}$. Puls 70; Atmung 12 .

Stets noch fester Schlaf. Tier erwacht bei starkem Kneifen, schläft abel gleich wieder ein.

11,40 Uhr Puls 100; Atmung 15.

1,30 Uhr p. m. erwacht das Tier und verbringt die nächste Zeit in leichtem Halbschlafe.

2,30 Uhr kann es etwas schwankend gehen.

3,30 Uhr läuft es ganz gut, erseheint aber noch ziemlich träge.

\section{Versuch. Hund B. 19. I. $14.14,85 \mathrm{~kg}$ Gewicht.}

$8,56 \mathrm{Uhr}$ a. m. wieder $0,1 \mathrm{~g}$ Neuronal pro Kilogramm Gewicht, total also $1,485 \mathrm{~g}$ Neuronal. Das Tier legt sich wieder gleich hin und schläft nach 8 Minuten ein.

9,10 Uhr Puls 92; Atmung 15; reagiert nicht mehr auf Anrufen, wohl aber noch auf heftiges Anfassen. Kornealreflex vorhanden.

9,30 Uhr Puls 88; Atmung 15

10,20 Uhr Puls 84; Atmung 14; stets tiefer Schlaf.

11,10 Uhr Puls 80 ; Atmung 12

2,00 Uhr p. m. erwacht das Tier und ist bald wieder ganz munter.

Nachdem ich so ein klares Bild von der Normalwirkung der betreffenden Neuronaldosis gewonnen hatte, wurde nun ein Versuch mit derselben Dosis bei einem Aufregungszustande an demselben Hunde durchgeführt.

\section{Versuch. Hund. B. 22. I. 14. Gewicht $14,4 \mathrm{~kg}$.}

9,05 Uhr a. m. erhielt das Tier eine subkutane Injektion von $0,1 \mathrm{~g}$ des Monomethylderivats des ac- $\beta$-T. 4 Minuten nach der Injektion setzt die Wirkung ein. Das Tier wird immer unruhiger, schnüfelt herum und winselt, bellt auch zuweilen ganz unmotiviert, was alles deutlich anf Halluzinationen hinweist. Die Pupillen sind maximal erweitert, Atmung und Herzaktion anßerordentlich beschleunigt.

9,15 Uhr. Bei voller Ausbildung des Aufregungszustandes erhält das Tier die übliche Neuronaldosis, $0,1 \mathrm{~g}$ pro Kilogramm Gewicht, total also $1,44 \mathrm{~g}$.

9,25 Uhr. Die Aufregung dauert in nnverminderter Stärke an, trotżdem das Tier sonst nach dieser Zeit schon schlief.

9,35 Chr. Zustand ist unverändert. Es ist wegen der steten motomotorischen Unruhe nicht möglich, Puls und Atmung zu zählen. 


\section{9,45 Uhr. Zustand unverändert:}

10,00 Uhr. Die Aufregung dauert, von einigen rubigeren Intervallen unterbrochen, immer noch an. Jetzt kann man aber deutlich die Koordinationsstörungen konstatieren, besonders an den hinteren Extremitäten. Diese Bewegungshemmung steigert sichtlich die psychisebe Aufregung des Tieres.

10,45 Uhr. Bewegungsstörung wird deutlicher.

11,00 Uhr. Das Tier wälzt sich winselnd auf dem Boden herrm es ist nicht fähig, sich anfzurichten.

11,30 Uhr das nämliche Bild.

3 Chr p. m. Das Tier ist etwas ruhiger geworden, doch sind die Pupillen noch stark erweitert, die Ataxie dauert an.

4 Uhr. Zustand unverändert.

5 Uhr hat endlich die Unruhe bedeutend nachgelassen, ebenso die Bewegungsstörungen. Die Pupillen sind jedoch noch erweitert.

6 Uhr. Das Tier ist ziemlich ruhig und kann, noch etwas schwankend, gehen.

Jedenfalls hatte dieser Versuch im Vergleich zu denen am normalen Tiere mit aller Deutlichkeit ergeben, daß der künstlich verursachte Aufregungszustand die bypnotische Wirkung der sonst geniigenden Neuronaldosis fast völlig aufhob, wie dies anch beim Menschen der Fall zu sein pflegt. Es fragt sich nun, wie in quantitativer Hinsicht die Mengen des Narkotikums im Gehirn bei beiden Zuständen sich verhalten. Daß wegen des Aufregungszustandes gar kein Nerronal ins Gehirn eindringe, ist wenig wahrscheinlich. Die dentliche Bewegungsstörung, welche ich bei dem Tier beobachtete, weist unbedingt auf eine narkotische Einwirkung auf die Bewegungszentren hin, was natïrlich nur durch ein Eindringen des Narkotikums in die Gehirnsubstanz bewirkt werden kann. Anf Grund aller der vorausgehenden Beobachtungen an diesem Tiere wurde nun der analytische Versuch ausgeführt.

\section{Versuch. Hund B. 31. I. 14. Gewicht $13,6 \mathrm{~kg}$.}

$8,40 \mathrm{Uhr}$ a. $\mathrm{m}$. sabkutane Injektion von $0,1 \mathrm{~g}$ des Monomethylderivats des $\beta-T$. Die Aufregung setzt wieder bald ein und bietet das nämliche Bild wie beim vorhergehenden Versuch.

8,56 Uhr. $0,1 \mathrm{~g}$ Neuronal pro Kilogramm, total also $1,36 \mathrm{~g}$.

9,16 Uhr. Die Aufregung dauert unverändert an, es stellen sich auch diesmal wieder die Koordinationsstörungen ein. Eine schlafmachende Wirkung des Neuronals bleibt wieder vollständig aus.

11,00 Uhr wird das Tier getötet.

Die quantitative Analyse der Organe von Hund B ergab: Gewicht des Gehirns $89 \mathrm{~g}$; Analyse verunglückt.

In $100 \mathrm{ccm}$ Blut gefunden $43 \mathrm{mg}$ Brom; anf $1050 \mathrm{ccm}$ berechnet $455 \mathrm{mg}$. In Prozenten des eingeführten Nerronals $75 \%$. 
In der Leber $83 \mathrm{mg}$ Brom; in Prozenten des eingeführten Neuronals $15 \%$.

In Magen und Darm $58 \mathrm{mg}$ Brom; in Prozenten des eingeführten Neuronals $10 \%$.

1. Versuch. Hund C. 13. I. 14. Gewicht 20,4 kg.

Puls 84; Atmung 25.

8,56 Uhr a. m. $0,1 \mathrm{~g}$ Neuronol pro Kilogramm Gewicht, total 2,04 g.

5-6 Minuten nachher leichte Unruhe, schwankender Gang, nach 8 Minuten legt sich das Tier hin und ist nicht mehr imstande, sich aufzurichten, es kämpft aber ganz intensiv und zuerst mit Erfolg gegen den Schlaf. Puls 84-92: Atmung 24.

11,00 Uhr schläft es aber schließlich doch ein, reagiert aber doch noch auf Anrufen und Stechen. Dieser leichte Schlaf danert noch um

3,00 Uhr p. m. an; Puls 100; Atmung 15.

7,00 Uhr ist das Tier ganz munter.

Dieses abweichende Ergebnis rührt daher, daß es sich um ein sehr mißtrauisches, frisch angekommenes Tier handelte. Wie der folgende Versuch beweist, reagiert auch dieses Tier in normaler Weise, nachdem es sich etwas an die Umgebung gewöhnt hat.

2. Versuch. Hund C. 21. I. 14. Gewicht $21,1 \mathrm{~kg}$.

9,05 Uhr a. m. 0,1 g Neuronal pro Kilogramm Gewieht, total also $2,11 \mathrm{~g}$.

9,20 Uhr ist das Tier eingeschlafen, reagiert aber noch anf Anrufen.

9,30 Uhr. Puls 76; Atmung 16; leichter Schlaf.

10,00 Uhr. » $70 ;$; 16 ; »

$10,30 \mathrm{Whr}$ leichter Schlaf.

$12,00 \mathrm{Vhr}$ ",

2,00 Uhr p. m. ist das Tier wieder wach.

2,30 Uhr kann es ziemlich gut gehen, ist aber anscheinend noch schläfrig, legt sich hin und schläft bald wieder fest ein.

3,30 Uhr. Puls 60; Atmung 12;

5,30 Uhr ist das Tier wieder munter.

3. Versuch. 23. I. 14 . Gewicht $20,8 \mathrm{~kg}$.

$8,52 \mathrm{Uhr}$ a. m. subkutane Injektion von $0,1 \mathrm{~g}$ der Base des ac-Tetrahydro- $\beta$-Naphthylamin. Nach etwa $1 / 4$ Stunde stellen sich die Zeichen einer leichten Erregung ein. Die Pupille erweitert sich und die Atmung wird beschleunigt, da jedoch die Dosis nicht zu genügen scheint, erhält des Tier'

9,22 $\mathrm{Uhr}$ eine weitere Injektion, diesmal $0,025 \mathrm{~g}$ der Base. erweitert.

9,45 Uhr. Die Aufregnng ist bedeutend heftiger, die Pupille stark

10,00 Uhr. $0,1 \mathrm{~g}$ Neuronal pro Kilogramm, total $2,1 \mathrm{~g}$. Der Erregungszustand steigert sich zusehends ind bietet im wesentlichen das gleiche Bild wie beim entspreehenden Versuch mit Hund B. 
Wirkg. d. Hypnotika (Neuronal) b. normal. u. b. psyehisch erregten Zuständen. 171

10,20 Ubr. $\begin{gathered}\text { Heftige Aufregung, es ist nicht möglich, Atmung und } \\ \text { Pulse zu zählen. Die Pupille ist maximal erweitert. }\end{gathered}$ Die Erregung steigert sich zusehends, dazu kommt die 11,00 Uhr. $\left\{\begin{array}{l}\text { Bewegungsstörung, durch welche das Tier noch mehr } \\ \text { gereizt wird. }\end{array}\right.$

12,00 Uhr. Heulend wälzt sich das Tier im Käfig herum, so daß man genötigt ist, es dureh Chloralhydrat zu beruhigen. Die durch das letztere Mittel schließlich erzengte tiefe Narkose dauert den ganzen Nachmittag an, doch tritt leider abends 9 Uhr Exitus ein.

Die quantitative Analyse an Hnod C ergab:

Gewicht des Gehirns $80 \mathrm{~g}$; Bromgehalt $9 \mathrm{mg}$; auf $100 \mathrm{~g}$ ber. $11 \mathrm{mg}$. In Prozenten des eingefülurten Neuronals $0,96 \%$; auf $100 \mathrm{~g}$ ber. $1,2 \%$.

Das Blut und die ïbrigen Organe konnten begreiflicherweise nicht analysiert werden.

\section{Versuch. Hand D. 29. I. 14. Gewicht 9,3 kg.}

$8,40 \mathrm{Uhr}$ a. m. $0,1 \mathrm{~g}$ Neuronal pro Kilogramm Gewicht, total 0,93 g. Wie gewöhnlich stellt sich nach einigen Minuten das Schwanken ein, begleitet von deutlichem Schlafbedürfnis.

9,15 Uhr schläft das Tier fest; Atmung 18; Puls 100.

9,30 Uhr Atmung 16; Puls 100; leichter Schlaf.

$10,00 \mathrm{Uhr}$ leichter Schlaf.

10,30 Uhr ist das Tier wieder wach und ganz munter. Von der Bewegungsstörung ist kaum mehr etwas zu bemerken.

Da die gewöhnliche Dosis von $0,1 \mathrm{~g}$ pro Kilogramm bei diesem anscheinend recht intelligenten und wachsamen Tiere nicht genügte, erhielt es beim

$$
\text { 2. Versuch, 4. II, } 14
$$

8,55 Uhr a. m. $0,15 \mathrm{~g}$ Neuronol pro Kilogramm, total bei einem Gewicht von $9,7 \mathrm{~kg} 1,45 \mathrm{~g}$. Das Tier wehrt sich wieder eine Zeitlang, legt sich dann aber nieder und ist

9,25 Uhr fest eingeschlafen. Es reagiert bald nicht mehr auf Anrnfen und Anfassen. Nach einiger Zeit jedoch stellt sich wieder das Zittern der Extremitäten ein, wie bei den hohen Dosen der anderen Tiere.

12,00 Uhr noch fester Schlaf.

$3,00 \mathrm{Uhr}$ p. m erwacht es und ist nach 1 Stunde ganz munter.

\section{Versueh. 13. II. 14. Gewicht $9 \mathrm{~kg}$.}

Der Verlauf des vorhergehenden Versuches genügte zur Feststellung der Schlafdauer und Intensität, und so folgte der definitive Versuch mit dem Aufregungsmittel.

3,15 Uhr p. m. 4 cem $(0,08 \mathrm{~g})$ des Monomethyls des $\beta$-T. subkutan. Die Aufregung setzt bald ein und bietet genan dasselbe Bild wie bei den anderen Tieren, ist jedoch nicht so heftig.

$3,30 \mathrm{Uhr} 0,15 \mathrm{~g}$ Neuronal pro Kilogramm, total also $1,35 \mathrm{~g}$. $\mathbf{A b}-$ gesehen von der Bewegungshemmung kommt keine andere Wirkung des 
Neuronals zur Geltung, und so kann das Tier, wie gewohnt naelz 2 Stunden durch Eutbluten getötet werden.

Die quantitative Analyse der Organe von Hund D ergab:

Gewicht des Gehirns $68 \mathrm{~g}$; Bromgehalt $8,12 \mathrm{mg}$; anf $100 \mathrm{~g}$ berechnet $11,9 \mathrm{mg}$.

In Prozenten des eingefübrten Neuronals $1,4 \%$; auf $100 \mathrm{~g}$ berechnet $2,05 \%$.

In $100 \mathrm{ccm}$ Blut gefunden $66 \mathrm{mg}$ Brom; anf $700 \mathrm{ccm}$ Blut berechnet $470 \mathrm{mg}$.

In Prozenten des eingeführten Neuronals $77 \%$.

In der Leber $57 \mathrm{mg}$ Brom; in Prozenten des eingefïhrten Neuronals 10\%. nals $15 \%$.

In Magen and Darm $90 \mathrm{mg}$; in Prozenten des eingefíhrten Neuro-

Diese erste beim erregten Tier einwandfrei durchgeführte quantitative Analyse zeigt uns, daB die Nichtwirkung des Neuronals keincsfalls herribren kann von einem Feblen des Narkotikums in Gehirn. Die gefundene Menge ist sogar etwas gröber als die beim normalen Zustand angetroffene.

1. Versuch. Hand E. 30. I. 14. Gewicht $7,8 \mathrm{~kg}$.

$8,40 \mathrm{Uhr}$ a.m. $0,1 \mathrm{~g}$ Neuronal pro Kilogramm, total $0,78 \mathrm{~g}$. Die Koordinationsstörungen setzen schon nach 4 Minuten ein. Nach kurzem Kampfe legt sich das Tier hin und ist um

9,00 Uhr fost eingeschlafen.

9,20 Uhr tiefer Schlaf; Puls 80; Atmung 12. Dieser feste und regelmäßige Schlaf dauert bis 4,00 Uhr p. m.

Dieser Hund reagierte so prompt auf das Mittel, daß man nach Ablauf der bestimmten Frist zum definitiven Versuche schritt. Das Tier war als Kontrolltier bestimmt.

\section{Versuch. 4. II. 14. Gewicht $8 \mathrm{~kg}$.}

4,00 Uhr p. m. $0,1 \mathrm{~g}$ Neuronal pro Kilogramm, total $0,8 \mathrm{~g}$.

4,15 Uhr schläft das Tiel ein und liegt die nächsten 2 Stunden in tiefem Schlafe.

6,00 Uhr durch Entbluten getötet.

Die Analyse der Organe von Hund E ergab: $9 \mathrm{mg}$.

Gewicht des Gehirns $71 \mathrm{~g}$; Bromgehalt $6,3 \mathrm{mg}$; anf $100 \mathrm{~g}$ berechnet

In Prozenten des eingeführten Neuronals $1,87 \%$; auf $100 \mathrm{~g}$ berechnet $2,6 \%$.

In $100 \mathrm{cem}$ Blut $40 \mathrm{mg}$ Brom; auf $600 \mathrm{ccm}$ Blat berechnet $240 \mathrm{mg}$ Bromgehalt.

In Prozenten des eingefübrten Neuronals $76 \%$. 
Wirkg.d.Hypnotika (Neuronal) b. normal. u. b. psychisch erregten Zustinden. 173

In der Leber $35 \mathrm{mg}$ Brom; in Prozenten des eingefïhrten Neuronols $12 \%$. In Magen und Darm nur Spuren.

Die analytischen Ergebnisse stimmen somit ziemlich gut mit denen der anderen Normalversuche bei einer Dosis von $0,1 \mathrm{~g}$ pro Kilogramm überein. Es wurde daher von einer weiteren Untersuchung: bei dieser Dosis abgesehen.

1. Versuch. Hund F. 30. 1. 14. Gewicht 9,9 kg.

8,48 Uhr a. m. 0,1 g Neuronol pro Kilogramm;

9,00 Uhr schläft das Tier schon fest. Dieser ruhige und tiefe Schlaf dáuert bis nachmittags gegen herum.

5,00 Uhr. Dann wird das Tier aber bald munter und läuft ganz gut

2. Versuch. 3. II. 14. Gewicht des Hundes 10,1 kg.

9,00 Uhr a. m. $0,1 \mathrm{~g}$ Neuronal pro Kilogramm.

9,10 Uhr schläft das Tier wieder fest. Der Schlaf dauert an bis 5,00 Uhr p. m, dann erholt sich das Tier sehr rasch.

Diese beiden Versuche decken sich so gut, daß am

10. II. 14 der definitive Versuch vorgenommen werden konnte.

3. Versuch. Gewicht des Tieres $9,7 \mathrm{~kg}$.

8,08 Uhr p. m. subkntane Injektion von $3 \mathrm{ccm}(0,06 \mathrm{~g})$ des Monomethyls des ac- $\beta$-T. Das Mittel wirkt, wie schon beschrieben, nach kurzer Zeit. Sobald die Aufregung einigermaßen ausgebildet ist, erhält der Hund

3,24 Uhr $0,1 \mathrm{~g}$ Neuronol pro Kilogramm. Der Verlauf ist der nämliche wie schon ausgeführt, Halluzinationen, Heulen und Umherwälzen, maximale Pupillenerweiterung, stark beschleunigte Atmung und Herzaktion. Die Aufregung wird auch hier wieder besonders durch die infolge del" Neuronalwirkung sich einstellenden Bewegungsstörungen genährt.

5,24 Uhr, also 2 Stunden später, wird das Tier durch Entbluten getötet.

Die quantitative Analyse der Organe von Hund $\mathrm{F}$ ergab: $10 \mathrm{mg}$.

Gewicht des Gehirns $70 \mathrm{~g} \cdot$ Bromgehalt $7 \mathrm{mg}$; auf $100 \mathrm{~g}$ berechnet $2,5 \%$.

In Prozenten des eingeführten Neuronals $1,75 \%$ auf $100 \mathrm{~g}$ berechnet

In $100 \mathrm{~g}$ cem Blut $41 \mathrm{mg}$ Brom; auf $750 \mathrm{ccm}$ berechnet $300 \mathrm{mg}$.

In Prozenten des eingefübrten Neuronals $78 \%$.

In der Leber $36 \mathrm{mg}$ Bromgehalt; in Prozenten des eingeführten Neuronals $10 \%$.

In Magen und Darm $30 \mathrm{mg}$ Brom; in Prozenten des eingeführten Neuronals $8 \%$. 
1. Versuch. Hund G. 7. II. 14. Gewicht 6,4 kg.

8,50 Uhr a. m. erhält das Tier $0,1 \mathrm{~g}$ Neuronal pro Kilogramm Gewicht. Es wurde von nun an auf die genaue Beobachtung von Atmung und Puls verzichtet, da sich dabei nichts Bemerkenswertes ergeben hatte. Das Augenmerk wurde nur auf Eintritt, Dater und Intensität des Schlafes gerichtet.

9,00 Uhr a. m. schläft das Tier ein.

$10,00 \mathrm{Uhr}$

12,00 Uhr tiefer Schlaf.

$2,00 \mathrm{Uhr}$

4,00 $\mathrm{Uhr}$ p. m. erwacht das Tier und hat sich nach einer Stunde ganz gut erholt.

2. Versuch. 11. II. 14. Gewicht 6,2 kg.

$8,30 \mathrm{Uhr}$ a. m. $0,1 \mathrm{~g}$ Neuronol pro Kilogramm.

$8,45 \mathrm{Uhr}$ ist das Tier fest eingeschlafen

$10,00 \mathrm{Uhr}$

$12,00 \mathrm{Chr}$ fester und ruhiger Schlaf.

2,00 Uhr

4,00 Uhr p. m. erwacht das Tier und ist bald wieder munter.

3. Versuch. 17. II. 14.

Dieser Hund war zum Kontrollhund bestimmt, nnd da uns die Wirkung bei einer Dosis von $0,1 \mathrm{~g}$ pro Kilogramm geklärt schien und auch die analytischen Daten hierfür bekannt waren, erhielt dieses Tier $0,15 \mathrm{~g}$ pro Kilogramm Gewicht. Es wurde dabei der Zweck verfolgt, zu untersuchen, ob unter diesen Umständen anch eine größere Menge des Narkotikums ins Gehirn gelange.

3,30 Uhr p. m. $0,15 \mathrm{~g}$ pro Kilogramm Gewicht, total also bei einem Gewichte von $6,25 \mathrm{~kg} 0,94 \mathrm{~g}$ Neuronal.

$3,45 \mathrm{Uhr}$ ist das Tier fest eingeschlafen. Der Schlaf danert an.

5,30 Uhr durch Entblutung getötet.

Die quantitative Analyse der Organe von Hund G. ergab:

Gewicht des Gehirns $61 \mathrm{~g}$; Bromgehalt $5,8 \mathrm{mg}$; anf $100 \mathrm{~g}$ berechnet $9,5 \mathrm{mg}$. $2,4 \%$.

In Prozenten des eingeführten Neuronals $1,48 \%$; anf $100 \mathrm{~g}$ berechnet

In $100 \mathrm{ccm}$ Blut gefunden $52 \mathrm{mg}$ Brom; auf $500 \mathrm{ccm}$ berechnet $260 \mathrm{mg}$ Bromgehalt.

In Prozenten des eingeführten Neuronals $70 \%$.

In der Leber $90 \mathrm{mg}$ Brom; in Prozenten des eingeführten Neuronals $20 \%$.

In Magen und Darm $40 \mathrm{mg}$ Brom; in Prozent des eingefiihrten Neuronals $9 \%$.

1. Versueh. Hund H. 7. II. 14 . Gewicht $6,3 \mathrm{~kg}$.

$8,55 \mathrm{Uhr}$ a. $\mathrm{m} .0,1 \mathrm{~g}$ Nemronal pro Kilogramm. Auch dieses Tier wehrt sich zuerst heftig gegen den Schlaf. 
Wirkg.d. Hypnotika (Nerronal) b. normal. u. b. psychisch erregten Zuständen. 175

9,25 Uhr ist es eingeschlafen.

10 Uhr fester Schlaf, der bis nachmittags

3 Uhr andauert, dann erwacht das Tier.

2. Versuch. 11. Il. 14. Gewicht $5,9 \mathrm{~kg}$.

8,28 Uhr a. m. $0,1 \mathrm{~g}$ Nenronal pro Kilogramm.

$8,50 \mathrm{Uhr}$ ist das Tier eingeschlafen. Der tiefe und ruhige Schlaf danert bis 1 Uhr p. m.

3 Ulr ist das Tier munter.

\section{Versuch. 20. II. 14. Gewicht $5,65 \mathrm{~kg}$.}

Dieses sonst schon sehr lebhafte und unruhige Tier schien mir geeignet für einen Kontrollversuch bei Aufregung. Bis jetzt waren diese Zustände durch chemisehe Mittel indirekt herbeigeführt worden. Es wurde deshalb hier versucht, durch direkte psychische Aufregung das Tier vom Neuronalschlaf abzuhaiten.

3,30 Chr p. m. $0,1 \mathrm{~g} \cdot$ Neuronal pro Kilogramm, total $=0,56 \mathrm{~g}$. Nin wurde das Tier' 2 Stunden lang unterhalten und am Schlafen verhindert. Es wurde ihm z. B. zeitweilig eine Katze in einem Käfig vorgeführt und dadurch das Tier ganz bedeutend erregt oder man setzte noch andere Tiere in den Käfig hinein. Durch das Zeigen einer schönen Wurst wurde seine Begierde genährt. Anßerdem führte man das Tier in den Laboratorien herum. Tatsächlich gelang es auf diese Weise, daß Tier psychisch so zu. erregen, daß der gewohnte Schlaf nicht eintrat, nur die motorischen Störungen stellten sich auch hier ein.

$5,30 \mathrm{Uhr}$ wurde es durch Entbluten getötet.

Die quantitative Analyse der Organe von Hund H ergab:

Gewicht des Gehirns $58 \mathrm{~g}$; Bromgehalt $5,91 \mathrm{mg}$; auf $100 \mathrm{~g}$ berechnet. 10,2 mg.

In Prozenten des eingefuhrten Neuronals $2,4 \%$; auf $100 \mathrm{~g}$ berechnet $4 \%$.

In $100 \mathrm{ccm}$ Blut gefunden $42 \mathrm{mg}$ Brom; anf $450 \mathrm{ccm}$ berechnet: $185 \mathrm{mg}$ Bromgehalt.

In Prozenten des eingeführten Neuronals $78 \%$.

In der Leber $40 \mathrm{mg}$ Brom; in Prozenten $15 \%$ des eingeführten. Neuronals.

Im Magen nur Spuren.

1. Versuch. Hand J. 20. IL. 14. 5,2 kg Gewicht.

$8,35 \mathrm{Uhr}$ a. m. $0,1 \mathrm{~g}$ Neuronal pro Kilogramm. Die gewöhnlichen Erscheinungen, wie Schwanken und große Schläfrigkeit, stellen sich bald. ein, doch ist das noch ganz junge Tier anfgeregt und die Umgebung ungewohnt.

9,45 Uhr schlaft das Tier jedoch ein.

10,45 Uhr elwacht es wieder und bleibt wach. 
2. Versuch. 21. M. 14. Gewicht $4,95 \mathrm{~kg}$. Kilogramm.

8,35 Uhr a. $\mathrm{m}$. erhält das Tier total $0,50 \mathrm{~g}$ Neuronal $=0,1$ pro

9,30 Uhr ist es fest eingeschlafen. Der ruhige schlaf dauert bis $12 \mathrm{Uhr}$ mittags, dann ist das Tier bald wieder munter.

Da dieser Hund zum Kontrolltier mit der größeren Dosis bestimmt war, warden diese zwei Vorversuche für genügend erachtet. Es folgte der

3. Versuch. 26. II. 14. Gewicht $4,95 \mathrm{~kg}$.

2,25 Uhr p. m. $0,15 \mathrm{~g}$ Neuronal pro Jilogramm, total also $0,75 \mathrm{~g}$. 2,35 Uhr schäft das Tier ein.

4,25 Uhr durch Entbluten getötet.

Die quantitative Analyse der Organe von Hund $J$ ergab: $9,8 \mathrm{mg}$.

Gewicht des Gehirns 70,5 g; Bromgehalt $6,8 \mathrm{mg}$; anf $100 \mathrm{~g}$ berechnet

In Prozenten des eingeführten Neuronals $2,22 \%$; auf $100 \mathrm{~g}$ berechnet $3,1 \%$.

In $100 \mathrm{ccm}$ Blut gefunden $71 \mathrm{mg}$ Brom; auf $380 \mathrm{ccm}$ berechnet $270 \mathrm{mg}$ Bromgehalt.

In Prozenten des eingeführten Neuronals $80 \%$.

In der Leber $20 \mathrm{mg}$ Brom; bzw. 15\% des eingefübrten Neuronals.

In Magen und Darm nur: Spuren.

\section{Versuch. Hund K. 25. II. 14. Gewicht $8,95 \mathrm{~kg}$.}

8,50 Chr a. m. $0,1 \mathrm{~g}$ Neuronal pro Kilogramm. Das Tier legt sich bald darauf nieder und schläft eir.

$9,10 \mathrm{Uhr}$ fester Schlaf. Dieser dauert mit kurzen Enterbrechungen bis gegen Mittag, doch ist das Tier dann noch nicht ganz munter.

2 Vhr p. m. ist das Tier vollständig wach und kann gut gehen.

2. Versueh. 2. III. 14. Gewicht $8,2 \mathrm{~kg}$.

11,50 Uhr a. $\mathrm{m}$. subkutane Injektion von $4 \mathrm{ccm}(0,04 \mathrm{~g})$ des Monomethyls von $\beta$. T. Die Aufregung bildet sich langsam aus.

$12,20 \mathrm{Uhr}$ erbält das Tier $0,15 \mathrm{~g}$ Neuronal pro Kilogramm, total also $1,23 \mathrm{~g}$, also die größere Dosis als Parallelversuch zı Versuch mit Hund D.

12,30 Uhr noch eine Injektion von $0,04 \mathrm{~g}$ Monomethyl. Die Erregung war nicht ganz so heftig wie in den ersten Fällen, bot aber das gleiche Bild, mit Halluzination, maximaler Pupillenerweiterung und besonders rapider Atmung und Herzaktion. Die Bewegungsstörung wurde sehr deutlich, von Schlaf war keine Spur.

2,20 Chr p. m. durch Entbluten getötet. 
Wirkg.d. Hypnotika (Neuronal) b. normal. u. b. psychisch erregten Zuständen. 177

Die quantitative Analyse der Organe bei Hund K ergab: $10,7 \mathrm{mg}$. $2,1 \%$.

Gewicht des Gehirns $65 \mathrm{~g}$; Bromgehalt $6,95 \mathrm{mg}$; auf $100 \mathrm{~g}$ berechnet

In Prozenten des eingeführten Neuronals $1,36 \%$; anf $100 \mathrm{~g}$ berechnet

In $100 \mathrm{ccm}$ Blut gefunden $57 \mathrm{mg}$ Brom; auf $640 \mathrm{cem}$ berechnet $370 \mathrm{mg}$ Bromgehalt.

In Prozenten des eingeführten Neuronals $75 \%$.

In der Leber $80 \mathrm{mg}$ Brom; bzw. 15\% des eingeführten Neuronals. In Magen und Darm $25 \mathrm{mg} ;$ bzw. $5 \%$ des eingeführten Neuronals.

1. Versuch. Hund L. 28. II. 14. Gewicht $18,2 \mathrm{~kg}$. $8,45 \mathrm{Uhr}$ a. m. $0,1 \mathrm{~g}$ Neuronal pro Kilogramm, total 1,82 g. 9 Uhr schläft das Tier fest ein.

12 Uhr fester Schlaf.

$3 \mathrm{Uhr}$ p. m. erwacht das Tier und erholt sich sehr rasch. Dieser Versuch wurde als Kontrolle genügend erachtet.

2. Versuch. 5. III. 14 . Gewicht des Tieres $18,5 \mathrm{~kg}$.

$2,25 \mathrm{Uhr}$ p. m. $5 \mathrm{ccm}(0,1 \mathrm{~g})$ des Monomethyls von $\beta$. T. subkutan injiziert. Da die Aufregung nicht stark genug erschien, folgte 2,45 Uhr eine weitere Injektion von $0,06 \mathrm{~g}$ des Monomethyls. Jetzt steigerte sich die Erregung sehr schnell, infolgedessen erhielt das Tier

2,55 Uhr $0,1 \mathrm{~g}$ Neuronal pro Kilogramm Gewicht, total bei der Größe des Tieres die beträchtliche Menge von 1,85 g. Der Aufregungszustand bietet das nämliche Bild wie bei den anderen Tieren, zunächst besonders starke Bewegungsstörung mit sehr heftiger Atmung und Herzaktion, und mit maximaler Pupillenerweiterung. Kein Schlaf.

4,55 Uhr dureh Entbluten getötet.

Die quantitative Analyse der Organe von Hund L ergab: $11 \mathrm{mg}$.

Gewicht des Gehirns $82 \mathrm{~g}$; Bromgehalt $9 \mathrm{mg}$; auf $100 \mathrm{~g}$ berechnet

In Prozenten des eingeführten Neuronals $1,17 \%$; auf $100 \mathrm{~g}$ berechnet $1,42 \%$.

In $100 \mathrm{ccm}$ Blut $45 \mathrm{mg}$ Brom; auf $1400 \mathrm{ccm}$ berechnet $630 \mathrm{mg}$ Bromgehalt.

In Prozenten des eingeführten Neuronals $80 \%$.

In der Leber $90 \mathrm{mg}$ Brom; bzw. 19\% des eingeführten Neuronals.

In Magen und Darm $30 \mathrm{mg}$; bzw. $3 \%$ des eingeführten Neuronals.

Gehen wir die aus vorstehenden Versuchen sich ergebenden Resultate durch, so können wir folgendes feststellen:

Eine Dosis von $0,1 \mathrm{~g}$ Neuronal pro Kilogramm Körpergewicht, auf den nüchternen Magen verabreicht, genügt, um Hunde für 4 bis 
7 Stunden in sehr deutliche Hypnose zu versetzen, Atmung nnd Puls sind dabei nicht wesentlich verändert, ebensowenig der Blutdruck. Die Sensibilität und ein Teil der Reflexe dagegen sind ziemlich herabgesetzt.

Durch größere Dosen, z. B. 0,25 g Nemronal und mehr pro Kilogramm Körpergewicht, werden die hypnotischen Erscheinungen entsprechend rertieft, und das Bild nähert sich der Narkose. Namentlich auffallend ist dabei ein starkes Zittern, das zeitweilig den ganzen Körper befällt. Seine Ursache ist zunächst noch unklar, doch werden ähnliche Erscheinungen beim Menschen beobachtet, und zwar nicht nur bei Neuronal. Bei solchen Tieren dauerte der Schlaf mitunter 60 Stunden an.

Die analytischen Untersuchungen haben ergeben, daß bei der gleichen Dosis von $0,1 \mathrm{~g}$ pro Kilogramm Körpergewicht anch eine auffallende Konstanz des Bromgehaltes im Gehirn vorhanden ist. Ich stelle hier die erhaltenen Zahlen nach zweistiindiger Einwirkung des Neuronals zusammen.

1. Normale Hunde.

a) Absolute Menge Neuronal im Gehirn berechnet aus Bromgehalt :

$\begin{array}{ccccc}\text { Hund A } & \text { Hund } \mathrm{E} & \text { Hund } \mathrm{G} & \text { Hund } \mathrm{J} & \text { Mittel } \\ 13,2 \mathrm{mg} & 15,1 \mathrm{mg} & 13,9 \mathrm{mg} & 16,3 \mathrm{mg} & 14,62 \mathrm{mg}\end{array}$

b) Relative Menge Neuronal auf $100 \mathrm{~g}$ Gehirnsubstanz berechnet:

$\begin{array}{rrrrr}\text { Hund } \mathrm{A} & \text { Hund } \mathrm{E} & \text { Hund } \mathrm{G} & \text { Hund } \mathrm{J} & \text { Mittel } \\ 20,3 \mathrm{mg} & 21,26 \mathrm{mg} & 22,8 \mathrm{mg} & 28,1 \mathrm{mg} & 23,11 \mathrm{mg}\end{array}$

c) Verabreichte Menge Neuronal pro Kilogramm Tiergewicht:

$\begin{array}{llllr}\text { Hund A } & \text { Hund } \mathrm{E} & \text { Hund } \mathrm{G} & \text { Hund J } & \text { Mittel } \\ 100 \mathrm{mg} & 100 \mathrm{mg} & 150 \mathrm{mg} & 150 \mathrm{mg} & 125 \mathrm{mg}\end{array}$

Aus Tabelle a), b), c) ergibt sich, daß bei gleicher Versuchsanordnung die Menge des im Gehirn vorhandenen Neuronals eine recht konstante ist. Fast noch mehr auffallend als diese Konstanz. ist der geringe Prozentsatz und die Kleinheit der absoluten Menge. Ich hatte ganz andere Zahlen erwartet, spricht man doch so viel von der Lipotropie der Schlafmittel, so daB man auch erwarten sollte, dafuir einen deutlichen quantitativen Ausweis zu erhalten. Statt dessen geht aus Tabelle d) und e) hervor, daß von einer ganz besonderen Anziehungskraft des Gehirns im Vergleich zu den übrigen. Geweben nicht wohl gesprochen werden kann. 
Wirkg.d. Hypnotika (Neuronal) b. normal. u. b. psychisch erregten Zuständen. 179

d) Prozentuales Verhältnis des Gehirngewichtes zum Tiergewicht:

$\begin{array}{ccccc}\text { Hund A } & \text { Hund E } & \text { Hund G } & \text { Hund J } & \text { Mittel } \\ \mathbf{1} \% & 0,88 \% & 0,97 \% & \mathbf{1 , 4 6 \%} \% & 1,077 \%\end{array}$

e) Prozentuales Verhältnis des im Gehirn gefundenen zum gesamten resorbierten Neuronal:

$\begin{array}{ccccc}\text { Hund A } & \text { Hund E } & \text { Hund G } & \text { Hund J } & \text { Mittel } \\ 2,44 \% & 1,88 \% & 1,65 \% & 2,20 \% & 2,04 \%\end{array}$

f) Verhältnis von d) zu e):

$\begin{array}{ccccc}\text { Hund A } & \text { Hund } \mathrm{E} & \text { Hund } \mathrm{G} & \text { Hund J } & \text { Mittel } \\ 1: 2,44 \% & 1: 2,13 \% & 1: 1,70 \% & 1: 1,50 \% & 1: 1,93 \%\end{array}$

Vergleicht man nun die Tabellen e) und d) miteinander und berücksichtigt dabei noch die für die Adsorption von Neuronal nicht in Betracht kommenden Gewebsteile, wie Knochen und Haut, ferner Magen-, Darm- und Blaseninhalt, so fallen die Werte für die Anziehungskraft des Gehirns (in der Tabelle f durch das Verhältnis d zu e ausgedrückt) noch niedriger ans. Da ich auf die Ausarbeitung der analytischen Technik ganz besondere Sorgfalt verwendet hatte, so kann es sich nicht um eine Täuschung handeln. Wir werden ans auf Grund dieser quantitativen Ergebnisse in Zukunft daran gewöhnen müssen, das Wesen der Narkose mehr in einer besonderen qualitativen Empfindlichkeit des Gehiras für die betreffenden Substanzen als in einem quantitativen Überwiegen der letzteren im Zentralnervensystem zu suchen. Dementsprechend haben auch meine Versuche ergeben, daß keine einfache Proportionalität besteht in dem Sinne, daß bei doppelter Intensität der Wirkung auch eine doppelte Menge des Hypnotikums im Gehirn gefunden wird. Es scheint mir vielmehr die Intensität der Wirkung proportional zu sein der Wurzel aus den betreffenden Substanzmengen. Es müßten jedoch in dieser Richtung noch weit mehr Versuche, und zwar mit verschiedenen Hypnoticis ansgeführt werden, um zur Aufstellung eines bestimmten Gesetzes gelangen zu können.

So weit, was die Verhältnisse bei normalen Tieren anbetrifft.

Bei den künstlich herbeigeführten Aufregungszuständen haben die sonst gut wirksamen Neuronaldosen in bezug auf den Eintritt des Schlafes vollkommen versagt. Immer deutlich vorhanden war jedoch eine durch das Neuronal verursachte motorische Störung. Durch diese Erscheinung wird von vornherein die Annahme hinfällig, es sei die verminderte Wirksamkeit der Hypnotika beim Erregungszostand auf eine Behinderung ihres Eindringens in die Ge- 
hirnsubstanz zurückzuführen. Des genaueren aber wird diese Annahme durch die bei den erregten Tieren erhaltenen Analysenresultate widerlegt.

2. A ufgeregte Hunde.

a) Absolute Menge Neuronal im Gehirn, berechnet aus Bromgehalt:

Hund $\mathrm{C}$ Hund $\mathrm{D}$ Hund $\mathrm{F}$ Hund $\mathrm{H}$ Hund $\mathrm{K}$ Hund $\mathrm{L}$ Mittel $21,6 \mathrm{mg} \quad 19,5 \mathrm{mg} \quad 17,0 \mathrm{mg} \quad 14,2 \mathrm{mg} \quad 16,7 \mathrm{mg} \quad 21,6 \mathrm{mg} \quad 18,43 \mathrm{mg}$

b) Relative Menge Neuronal auf $100 \mathrm{~g}$ Gehirnsubstanz berechnet:

Hund $\mathrm{C}$ Hund $\mathrm{D}$ Hund $\mathrm{F}$ Hund $\mathrm{H}$ Fund $\mathrm{K}$ Hund $\mathrm{L}$ Mittel $27,25 \mathrm{mg} \quad 28,3 \mathrm{mg}, 24,3 \mathrm{mg} \quad 24,5 \mathrm{mg} \quad 25,7 \mathrm{mg} \quad 26,3 \mathrm{mg} \quad 26,06 \mathrm{mg}$

c) Verabreichte Menge Neuronal pro Kilogramm Tiergewicht: Hund $C$ Hund D Hund F Hund $H$ Hund $K$ Hund L Mittel $100 \mathrm{mg} \quad 150 \mathrm{mg} \quad 100 \mathrm{mg} \quad 100 \mathrm{mg} \quad 150 \mathrm{mg} \quad 100 \mathrm{mg} \quad 116,6 \mathrm{mg}$

Es ergibt sich aus dieser Tabelle im Vergleich mit der ersten, daß im Aufregungszustand sogar eine größere Menge des Hypnotikums den Weg in die Gehirnsubstanz findet als beim normalen. Auch hier sind die Werte ziemlich konstant, trotz der ganz verschiedenen Hirngewichte, der verschiedenen Größe der Tiere und dementsprechend anch der Verschiedenheit der angewandten absoluten Dosis. Der beim Aufregungszustand gefundene Mehrbetrag im Gehirn findet wohl seine beste Erklärung durch die Annabme, daß das Gehirn der erregten Tiere funktionell mehr leistete als das der normalen, und daß es infolgedessen auch mehr Blut an sich zog und mit dem Blute selbstrerständlich auch eine größere Menge des Narkotikums. Das scheint mir die einfachste Erklärung. Sie fußt auf der Tatsache, daß das Gehirn sich nicht als ein Ort mit besonderer quantitativer Anziehungskraft für das Narkotikum erwiesen hat, sondern daß die prozentuale Verteilung des Schlafmittels auf dasselbe sicher parallel geht dem prozentnalen Anteil des Gehirns am Körpergewicht nach Abzug der nicht funktionierenden Gewebsteile. Dies sei auch hier durch die Tabellen d), e) und f) illustriert.

d) Prozentuales Verhältnis des Gehirngewichtes zum Tiergewicht:

$\begin{array}{ccccccc}\text { Hund C } & \text { Hund D } & \text { Hund F } & \text { Hund H } & \text { Hund K } & \text { Hund L } & \text { Mittel } \\ 0,38 \% & 0,75 \% & 0,72 \% & 0,92 \% & 0,79 \% & 0,44 \% & 0,66 \%\end{array}$

e) Prozentuales Verhältnis des im Gehirn gefundenen Neuronals zum resorbierten:

$\begin{array}{llllllr}\text { Hund C } & \text { Hund D } & \text { Hund F } & \text { Hund H } & \text { Hund K } & \text { Hund L } & \text { Mittel } \\ 1,08 \% & 1,77 \% & 1,81 \% & 2,53 \% & 1,42 \% & 1,22 \% & 1,64 \%\end{array}$


Wirkg. d. Hypnotika (Neuronal) b. normal. u. b. psychisch erregten Zuständen. 181

f) Verhältnis von d) zu e):

Hund C Hund D Hund F Hund H Hund $\mathrm{K}$ Hund L Mittel

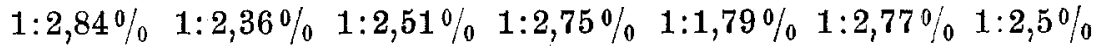

Betrachten wir das Verhältnis der Werte für die Anziehungskraft des Gehirns im normalen und im erregten Zustande zueinander, so finden wir dies durch die Zahlen 1,93:2,5 ausgedrückt.

Wenn daher bei Erregungszuständen eine größere Menge Neuronal im Gehirn gefunden wird, so kann dies meines Erachtens nur einen Beweis dafür abgeben, daß der Erregungszustand verbunden ist mit einer besseren Durchblutung, mit einer aktiven Hyperänie im Gehirn. Es bedingt somit der Übergang vom normalen zum Erregungszustand der Gehirnzellen durchaus keine Verschiebung des Teilungskoeffizienten für die Narkotika. Wenn aber die chemischen Verhältnisse bezüglich Adsorption, Durchlässigkeit usw. bei den normalen und erregten Gehirnzellen die gleichen bleiben, die Wirksamkeit der Narkotika aber beim Erregungszustand völlig versagt, so ergibt sich als Konsequenz die Auffassung; daß der Aufregungszustand biologisch einfach der Antagonist der Hypnose ist, und zwar im Sinne einer physiologisehen Mehrleistung des funktionierenden Protoplasmas. Man darf demnach auch eine gewisse Proportionalität erwarten zwischen Intensität der Aufregung und Höhe der zu ihrer Beseitigung nötigen Dosierung. Dies ist in der Praxis bereits nachgewiesen worden. Für jeden Erregungszustand gibt es eine bestimmte Dosis eines Narkotikums, welche im Blute die für den Zustand. der Mehrleistung des Zentralnervensystems notwendige toxische Grenzkonzentration schafft. Die dadurch erzielten Wirkungen gleichen schließlich denen bei normalen Individuen, jedoch ist die Dosis zur Erreichung dieser Wirkungen auf ein höheres Niveau eingestellt. Folgerichtig kann es dabei vorkommen, daß bei ungleichmäBiger Verteilung der Erregnngszustände einzelne Gegenden des Gehirns durch das Narkotikum stärker beeinflußt werden als andere; darauf beruhen die verschiedenen Wirkungen auf motorische und psychische Funktionen, wie sie sich bei unseren Versuchen ergeben haben. Dies bedeutet unter Umständen eine gewisse Gefahr für Zustände mit Dissoziation der Aufregung, z. B. zwischen Großhirnrinde und Medulla oblongata, da eine der erhöhten Funktion angepaßte relative Überdosierung des Narkotikums einer absoluten Überdosierung für einen anderen .Ort mit verminderter Erregung gleichkommt. 


\section{Zusammenfassung.}

Eine Dosis von $0,1 \mathrm{~g}$ Neuronal pro Kilogramm Körpergewicht genligt, um bei Hunden einen ruhigen Schlaf von 4-7 Stunden zu erzeugen.

Durch größere Dosen, z. B. 0,25 g Neuronal pro Kilogramm Gewicht, wird die Hypnose entsprechend vertieft, das Bild nähert sich der Narkose.

Die Analyse des entbluteten Gehirns, stets 2 Stunden nach der Eingabe des Mittels entnommen, ergab eine auffallende Konstanz des Neuronalgehaltes. Dieser schwankte bei normalen Hunden zwischen 13,2 und $16,3 \mathrm{mg}$ pro Gehirn bzw. 20,3 und $28,1 \mathrm{mg}$ auf $100 \mathrm{~g}$ pro Gehirnsubstanz. Mit Rücksicht auf die Größe der verabreichten Dosis erscheint dieser Wert als ein sehr geringer.

Bei den künstlich erzeugten Aufregungszuständen versagte die hypnotische Wirkung der gewohnten Neuronaldosen vollständig. Deutlich festzustellen war dagegen immer eine motorische Störung als Folge der Neuronaldarreichung.

Die quantitativen Analysen ergaben bei den Aufregungszuständen sogar höhere Werte für Neuronal im Gehirn als bei den normalen Tieren, sie schwankten hier zwischen 14,2 und $21,6 \mathrm{mg}$ pro Gehirn bzw. 24,3 und 28,3 $\mathrm{mg}$ auf $100 \mathrm{~g}$ Hirnsubstanz. Das Verhältnis der Anziehungskraft des Gehirns im normalen und im erregten Zustande für Neuronal wird durch die Zahlen 1,93:2,5 ansgedrückt.

Der Aufregungszustand verringert somit die Permeabilität der nervösen Membranen für die Schlafmittel nicht; die Wirkungslosigkeit derselben bei Erregung ist nicht durch ein geringeres Eindringen der Hypnotica zu erklären. Demnach ist der Anfregungszustand als der funktionelle Antagonist der Hypnose aufzufassen; zur Beseitigung der funktionellen Mehrleistung braucht es eine entsprechend höhere Konzentration des Hypnotikums im Blate. 